\title{
Die Unlust am Text
}

Notizen über eine Kehrseite und ihre Konsequenzen

Andreas Mauz

$» \mathrm{~N}$... fand einen vorzüglichen Gefallen daran, Klopstocks Messiade Reisern ganz vorzulesen;

bei der entsetzlichen Langenweile nun, die diese Lektüre beiden verursachte, und die sie sich doch einander, und jeder sich selber kaum zu gestehen wagten, hatte N... doch noch den Vorteil des lauten Lesens, womit ihm die Zeit verging: Reiser aber war verdammt zu hören, und über das Gehörte entzückt zu sein ...«. Karl Philipp Moritz, Anton Reiser (1785-90)

\section{I.}

Wer dies liest, ist doof. Ein ärgerlicher Satz. Wehrlos ist man ihm ausgeliefert, denn die Falle schnappt zu, bevor sie zu erkennen ist. Aber genau dies macht den Satz aus, macht ihn nicht nur strukturell interessant, sondern auch - und das möchte er wohl sein - amüsant. Ein Scherz, nicht besonders lustig, aber doch trickreich; man wird ihm seine `Lehre〈 nicht zu übel nehmen, es ist ja nur ein Satz. Ärgerlicher aber ist es, wenn man sich nach der Lektüre von einigen hundert Seiten eingestehen muss, dass der Text dies zwar nicht explizit gesagt hat, dass aber genau das der Fall ist: Wer dies liest bzw. gelesen hat, ist doof. Die Lektüre - d. h. der Text und dessen Rezeption hat die Erwartungen, die sich mit ihr verbanden, enttäuscht, sie gibt nicht her, was man sich von ihr erhoffte, was immer das im Einzelnen auch war. Damit ist ein strukturelles Problem benannt, das Leser/innen nur zu vertraut ist: Lesen ist ein Vollzug mit offenem Ausgang. Worauf man sich mit einem bestimmten Text eingelassen hat, weiss man im Einzelnen immer erst im Nachhinein, und eben deshalb ist man als Leser/in zwangsläufig immer wieder >doof gelesen, was man, hätte man gewusst, womit man es zu tun hat, nicht gelesen hätte. Bereits aus diesem simplen Grund lässt sich nicht vermeiden, dass Leseerfahrungen immer wieder Unlusterfahrungen werden. - Ihnen, den negativen Affekten, die die Lust am Text mehr oder weniger aufdringlich stören, gelten die folgenden Überlegungen.

Zur Debatte steht also eine Kehrseite. Denn der Hintergrund, vor dem das Leseleid $\mathrm{zu}$ profilieren ist, ist das weit verbreitetere Leseglück. So wenigstens stellt es sich dar, nimmt man die entsprechende Literatur zur Kenntnis (Anthologien mit Leseerlebnissen, die >Was liest ...<-Seite des Magazins `Literaturen` und dergleichen). Kaum ein 
autobiographisches Zeugnis, das auf ein Lob des Lesens, auf die Darstellung - insbesondere kindlicher - Glückserfahrungen beim Lesen verzichtet. Die Unlust am Text, scheint es, ist die seltene und selten thematisierte Ausnahme, der Regelfall ist die Lust. Ihre Beschreibung erfolgt fast topisch durch stets wiederkehrende Motive und Begriffe: das Buch als Rückzugsort oder Gegenwelt, das >Eintauchen $<$ oder >Versinken $<$ in der Lektüre, das >Verschlingen $<$ eines Buches, von dem man ssich kaum losreissen das man sin einem Zuge < durchliest oder heimlich >unter der Bettdecke`, der Autor, nach dem man >süchtig` ist, etc. Festzustehen scheint: Wer liest, will Lust und bekommt sie auch. Daher der Wunsch nach der >unendlichen Geschichte`, daher der diesbezüglich einschlägige Vers Borges': »... ich, der ich mir so oft das Paradies / in Form einer Bibliothek vorstellte« (>Poema de los dones $/>$ Gedicht von den Gaben $)$.

Um eine der entsprechenden Darstellungen etwas ausführlicher zu zitieren, sie stammt aus Walsers (Martin, ausnahmsweise) Lebenslauf eines Lesers (1992):

»Der Großonkel hatte diese treuherzigen Schinken und ähnliche Meisterwerke der Biederkeit als Romanbibliothek - immer drei in einem Band meterbreit im glasbehüteten Schrank. Ich war dort im Freien, griff zu, schlug auf - und weg war ich. Nicht mehr im beschützenden Allgäu, sondern in jenen stahlstichverzierten Jahrhunderten, in denen dem Tugendhaften nur deshalb so schrecklich nachgestellt wird, daß man ihn dann um so wunderbarer retten kann. Da war ich also als Lesender weg, aber nachträglich weiß ich deutlicher, wo ich gewesen bin, als wenn ich nicht durch Lesen weg gewesen wäre. Das ist das Paradox der durch Lesen bewirkten Lebenssteigerung. Die mit Büchern verbrachte Zeit - das ist eine leuchtende Spur in der ins Dunkle tendierenden Vergangenheit. ... der Zehnjährige, der beim Kaplan des Nachbardorfes den nächsten Band Karl May holt und die vier Kilometer zurück eher rennt als geht, um endlich, endlich dieses dicke kleine Buch aufschlagen zu können. Die Glut der Vorfreude des längst infizierten Karl-May-Lesers hat das Zimmer in eine Unvergänglichkeitslicht getaucht. ... Wenn ich nicht aufpasse, verklären sich mir diese Lesestunden zu reinen Goldgrundbildern « 1 .

Dass derartige Zeugnisse - auch abzüglich der retrospektiven Stilisierung - eine Erfahrung beschreiben, die Leser/innen vertraut ist, steht ausser Frage. Wäre sie es nicht, sie würden nicht lesen. Und doch scheint die Akzentuierung des Leseglück die Kehrseite (wenn sie denn eine ist und nicht doch eher der Regelfall) vergessen zu machen: den Umstand, dass Lesen immer wieder eine Anstrengung ist, eine Zumutung, ein Ärgernis, dass Bücher überfordern, enttäuschen, langweilen, quälend sich hinziehen und nicht enden wollen kurz: dass sich beim Lesen immer wieder ein breites Spektrum ne-

${ }^{1}$ M. Walser, Aus dem Lebenslauf eines Lesers, in: ders., Vormittag eines Schriftstellers, Frankfurt a.M. 1994, 53f. 
gativer Affekte einstellt, die es wünschenswert machen, die Lektüre des betreffenden Textes nicht fortzusetzen. Sie, die gelegentlich nahe legen, sich auch die Hölle als Bibliothek vorzustellen, bilden den Anlass vorliegender bescheidener Gegenprobe.

II.

Man wird Differenzierungen fordern: Welcher Leser? Welches Lesen? Welche Bücher? Und ist das Raster Lust/Unlust nicht etwas grob? - Sicher, viele Unterscheidungen wären $\mathrm{zu}$ machen. Im gegebenen Zusammenhang ist das nur bedingt möglich, allerdings aber auch nicht nötig, da das Augenmerk weniger der Unlust selbst als vielmehr deren Konsequenzen gelten wird. Bezüglich der Fragen, die die Thematik als solche unweigerlich nach sich zieht, bleibt es daher bei rudimentären Andeutungen.

Zunächst die ebenso basale wie banale Feststellung: Gelesen wird Verschiedenstes, aus verschiedensten Gründen und in verschiedensten Kontexten. Das Lesen - und damit, als dessen Begleiterscheinung, auch die Unlust am Text - ist in hohem Masse abhängig von historisch variablen intentionalen und situativen Faktoren. Die Pluralität und Heterogenität der Leseszenen setzt sich fort in der Pluralität und Heterogenität der ihnen korrespondierenden Unlusterfahrungen. Die Weite, die sich hier andeutet, lässt noch einmal genauer nach dem >Lusttext $<$ fragen, der den Hintergrund des anvisierten Übels abgibt. Auch aus den Erinnerungen Walsers geht hervor: Sein Paradigma ist der epische Text - die Erzählung, der Roman -, in dessen abgeschotteter Welt allerlei tolle Dinge passieren, die den Leser "weg« holen, ins wilde Kurdistan, in "stahlstichverzierte Jahrhunderte«, wohin und in wen auch immer, ihn auf jeden Fall durch die Erfahrung einer »Lebenssteigerung« >infizieren`. Man sollte dies nicht voreilig als blosse Beschreibung kindlicher Karl May- bzw. Harry Potter-Lektüre abtun; es ist derselbe Reiz der eigengesetzlichen fiktiven Welten, dem auch der erwachsene (Harry Potter-) Leser erliegt. Auch er zieht seine Lust aus der Einlassung auf das Als$\mathrm{Ob}$, das der swirklichen Welt eine Vielzahl möglicher Welten relativierend entgegensetzt, seien sie nun phantastischer Art oder eher >mimetisch`, etwa die kalkuliert tragische, kalkuliert erotischhappyendliche Welt des Arztromans.

Da sich die Lust am Text aber nicht im Paradigma des Romans erschöpft, wäre ihren gattungsspezifischen Ausprägungen Rechnung zu tragen. Die (Un-)Lust am Roman ist eine andere als die am Gedicht, eine dritte ist die am Dramentext, die sich ihrerseits von der an der Inszenierung unterscheidet; sie alle erfreuen bzw. enttäuschen durch je spezifische Stärken und Schwächen. Deshalb lesen wir einmal das eine und einmal das andere oder eben nicht. 
Innerhalb der Vielfalt der Leseszenen ist das prototypische >professionelle Lesen`sogenannt sschöner Literatur`von besonderem Interesse. Der philologische Leser unterhält (im Gegensatz zum prototypischen Amateur-Leser, der in seiner Freizeit saus Lieber liest, dieselben oder andere Texte) ein in mehrfacher Hinsicht eigentümliches Verhältnis zur Lust und Unlust am Text. Er ist zunächst zu bedauern. Wenn nahe liegt, dass die unfreiwillige Lektüre anfälliger ist für Unlustregungen, dann ist er, der seinen Alltag zu einem wesentlichen Teil lesend verbringt, auch dann, wenn ihm nicht danach ist, nicht zu beneiden. Allerdings kommt die Unlust dem philologischen Lesen noch in anderer, grundsätzlicherer Weise in die Quere, insofern nämlich, als sie dessen Programmatik anhaftet. Der Literaturwissenschaftler kennt die Regeln des Spiels, spielt aber nicht mit, denn er erlangt die Erkenntnis eines Werks gerade, »indem [er] seiner unmittelbaren Wirkung widersteht ${ }^{2}$. Indem sein Lesen der Lust entsagt, wird es tendenziell unlustig. Wer sich im Rahmen eines Seminars schwärmerisch über seine Ergriffenheit durch einen Text verbreitet, macht sich des Laientums verdächtig, er wird belächelt; Bemerkungen zu negativen Leseerfahrungen sind, da sie als kritisch gelten dürfen, eher zulässig (nur unter dieser Voraussetzung ist die Jeremiade von der Philologie, die die Lust am Lesen austreibe, verständlich - wie auch deren intellektuelle Varianten, etwa Susan Sontags fulminantes Votum »against interpretation « und für eine »erotic of art«.) Die Absage an die Lust >erster Ordnungく schliesst allerdings nicht aus, dass literaturwissenschaftliches Lesen gerade darin eine Lust >zweiter Ordnung bereitet. »Eine Welt der Bedeutungen zu kennen, ohne je von ihr betroffen zu sein, macht das Glück des Interpreten aus $\ll^{3}$.

Was die Literaturwissenschaft in ihren Lektüren methodisch neutralisiert, interessiert sie aber auch als Affekt des Normallesers eher wenig. Die Leser, die in den Blick geraten, wenn von Lust und Unlust am Text die Rede ist, haben mit den heuristisch äusserst wertvollen rabstrakten Lesern`, die vor etwa 30 Jahren in Konstanz und andernorts entdeckt wurden - dem impliziten, dem informierten, dem idealen, dem Superreader etc. - wenig bis gar nichts zu tun. Legte die Rezeptionsästhetik gegen die Objektivitätsansprüche des Strukturalismus den Akzent auf die eminente Rolle des Lesers, in dessen Bewusstsein sich der Text allein plural konkretisiert, so folgte sie in ihrem Verständnis von Lektüre dennoch dem formalistisch-strukturalistischen Begriff des Textes bzw. dem hermeneutischen des Werks. Der Akt des Lesens richtet sich auf den integralen Text, in dieser Auffassung trifft sie sich mit dem Reader-Response

${ }^{2}$ H. Schlaffer, Poesie und Wissen. Die Entstehung des ästhetischen Bewußtseins und der philologischen Erkenntnis, Frankfurt a.M. 1990, 191.

3 Ebd., 219. 
Criticism, der historischen Lesersoziologie und der empirischen Leserpsychologie. Und eben dies ist, wie Heinz Schlaffer in einem Beitrag zum tatsächlichen Umgang des Lesers mit Literatur gezeigt hat, die Ausnahme: "Was als Normalfall literarischer Rezeption angesehen oder konstruiert wird, ist in Wahrheit der Sonderfall einer philologischen Pflicht «" Es geht also, um eine begriffliche Unterscheidung Schlaffers aufzunehmen, weniger um den Leser als um den Lesenden, um die Person, die "im Akt der Lektüre eines Textes begriffen ist $~^{5}$, anders als der Leser, der Leser bleibt auch wenn er gerade nicht liest, "sondern auf eine Vielzahl von Lektüren zurück- und vorausblickt « ${ }^{6}$. Erst die Orientierung am Lesenden zeigt die phänomenale Breite dessen, was `lesen genannt wird. Nur so wird deutlich, dass lesen "mit Handlungen und Haltungen einhergeht, die mitunter dem Nicht-Lesen ähnlicher sehen als dem Lesen «" Und darauf kommt es an, an sich, vor allem aber dann, wenn der Lesende Unlust verspürt.

III.

"Wenn so a bravs Mannsbild z'grund gehn muss, nacher wird's mir z'dumm! Nacher schreib ich dem Buchhändler in Innsbruck a Briefl! Der soll sich gfreun! Und's Geld muss er mir wieder zruckgeben. Für so was zahl ich net. Derschlagen und derstechen und betrügen und belügen tun sich d'Leut sowieso schon im Leben gnug. Was brauch ich denn da noch a Büchl dazu? Wenn ich a Büchl lies, möcht ich mei' Freud dran haben. Dass ich's ganze Sauleben drüber vergessen kann! Und's Herz muss mir sein, als hätt's a frischgwaschens Hemmed an und a Feiertagsgwandl! Sonst pfeiff ich auf die ganze Dichterei!«.

Ludwig Ganghofer, Das Schweigen im Walde (1899)

Das oben angedeutete landläufige Lob des Lesens suggeriert zweierlei. Nahegelegt wird nicht nur, dass Bücher im Normalfall nichts anderes sind als Lustspender (wobei das >Vergnügen an tragischen Gegenständen`, die Angstlust des Krimilesers etc. unbedingt einzuschliessen sind), sondern auch, dass dies so sein soll. Literatur ist und hat zu sein eine Unterhaltungsveranstaltung, ein schöner Zeitvertreib, oder, etwas theoretischer, ein Mittel, die Realität zu suspendieren, ein entlastendes Kompensat für das, was diese nicht bieten will. Sie wird zum konkreten Fall des allgemeinen anthropologischen Lustprinzips, demzufolge der Mensch sucht, was ihm Lust verschafft, und was zu Unlust führt, meidet. Diese Auffassung wurde

\footnotetext{
${ }^{4}$ H. Schlaffer, Der Umgang mit Literatur. Diesseits und jenseits der Lektüre, Poetica 31, 1999, $1-25,3$.

${ }^{5}$ Ebd., 2.

6 Ebd.

7 Ebd., 4.
} 
und wird nun nicht uneingeschränkt geteilt. Dass Literatur immer wieder Glücksgefühle bereitet, mag ja sein, dies im Sinne einer Sollen-Forderung auch zum Idealfall zu erklären, liegt deshalb noch nicht auf der Hand:

"Ich glaube, man sollte überhaupt nur solche Bücher lesen, die einen beißen und stechen. Wenn das Buch, das wir lesen, uns nicht mit einem Faustschlag auf den Schädel weckt, wozu lesen wir dann das Buch? Damit es uns glücklich macht, wie Du schreibst? Mein Gott, glücklich wären wir eben auch, wenn wir keine Bücher hätten, und solche Bücher, die uns glücklich machen, könnten wir zur Not auch selber schreiben. Wir brauchen aber die Bücher, die auf uns wirken wie ein Unglück, das uns sehr schmerzt, wie der Tod eines, den wir lieber hatten als uns, wie wenn wir in Wälder verstoßen würden, von allen Menschen weg, wie ein Selbstmord, ein Buch muß die Axt sein für das gefrorene Meer in uns. Das glaube ich".

Kafkas Absage an den Trost der Literatur - sie findet sich in seinem Brief an Oskar Pollak vom 27. Januar $1904^{8}$ - gilt Lesern und Autoren gleichermassen. Sie fordert eine >bessere Literatur, was nach seiner Auffassung bedeutet, dass sie in durchaus gewalttätiger Weise wirkt "wie ein Unglück«, und sie fordert einen Leser, der bereit ist, sich dieser Literatur auszusetzen. Denn nur solche Bücher lohnt es sich zu lesen, nur sie besitzen die Kraft, »die Axt zu sein für das gefrorene Meer in uns". Die horazische Doppelformel vom prodesse et delectare lautet in der Fassung Kafkas: prodesse et irritare (und einiges spricht dafür, dass er selbst dieses Programm auch eingelöst hat, mit Sicherheit seinen zweiten Teil). - Kafkas Forderungen lassen sich je auf wiederum grundlegend-trivale Sachverhalte hin entfalten.

(1) Die Opposition lustvoll/unlustvoll ist nicht mit der von >guter bzw. >schlechter Literatur zu parallelisieren. Kafka kann weh tun, während die Lektüre der Gedichte des »Schlesischen Schwans« Friederike Kempners, Inbegriff >schlechter Lyrik, als vergnüglich empfunden wird. Unlüste werden in bestimmten Fällen gerade zum Qualitätsmerkmal, etwa zum Anzeiger positiv bewerteter Schwierigkeit. Sie sind der Preis, den es sich lohnt zu zahlen; sie schrecken die Vielen, den Leserplebs, ab und machen das betreffende Buch zum Besitz eines exklusiven Kreises von Kenner/innen. Eine bildungsbürgerliche Form der Lektüre immunisiert sich gegen Unlustempfindungen, indem sie Lesen als auch leidvolle Leistung versteht oder gar als Opfer, das der Kultur geschuldet wird.

(2) Unlüste sind nicht zwingend Anzeichen einer missglückten im Sinne von: nicht vorgesehenen - Rezeption, sie können auch beabsichtigt sein. Kafka will weh tun. Unlust wird zum produktionsästhetischen Element, zu welchem Ende auch immer. Seit Aristoteles' Empfehlung (Poetik, Kap. 6), dem Zuschauer der Tragödie

${ }^{8}$ F. Kafka, Briefe 1902-1924, Frankfurt a.M. 1975, 27 . 
zum Zweck der Katharsis phobos und eleos, Schrecken und Jammer (oder mit Schwadewaldt: »Gänsehaut und nassen Taschentüchern«) auszusetzen, sind die negativen Affekte als intendierte in der Dichtungstheorie gegenwärtig. Zumal die Poetiken der Moderne experimentieren kalkuliert und offensiv mit dem ganzen Register der nicht mehr schönen Empfindungen. Das Lustbedürfnis des Lesers wird nicht mehr, nicht mehr so unmittelbar und nicht in der erwarteten Weise bedient; antimimetische und antiillusionistische Impulse brechen das Ideal des Lusttextes auf, die in sich geschlossene Textgegenwelt. Durch - pauschal gesprochen - »offene" oder "schreibbare« (Barthes) Texte wird eine zu schlichte Konsumhaltung zurückgewiesen. Das delectare wird erschwert, ganz einerlei, ob am prodesse festgehalten wird oder nicht. Darin kommen, um zur Abwechslung dramatische Beispiele zu wählen, Ionesco, der auf »das Unerträgliche" setzt, und die Beiss- und Stecheffekte des Brechtschen Epischen Theaters überein. Brecht verwirft das restlose Eintauchen in die "sanfte Narkose" (Freud) der Literatur (des Dramas) aus politisch-emanzipatorischem Interesse; verschiedenste Formen der Illusionsbrechung und Verfremdung zwingen den Zuschauer, wenigstens theoretisch, zu kritischer Reflexion. Dass solche Störungsmassnahmen bewusst eingebaut werden müssen, belegt aber nur, dass er an sich eher nicht auf politische Belehrung aus war, sondern, wie Oskar Pollak, auf »Glück«. Aus dem gleichen Grund waren und sind auch die Zumutungen des Absurden Dramas oder die Mysterienspiele Hermann Nitsch' nicht mehrheitsfähig; aus dem gleichen Grund verläuft parallel zu den Texten der Moderne auch ein breiter, akademisch weitgehend unbeachteter Strom `vormoderner`, >traditioneller`, >trivialer Literatur, die diesem Bedürfnis entgegenkam und kommt.

Aber: Die Wirkabsicht ist eine Sache, die Wirkung, die dieser permanent entläuft, eine andere. Brecht kann auch >kulinarisch szeniert und konsumiert werden; intendierte Regungen, lustig und unlustig, treten an falscher Stelle auf oder bleiben aus. Lesende lesen immer mehr oder weniger 'gegen den Strich`, oder genauer: gegen die Striche, wenigstens den des Autors, den anderer Lesender und den eigenen. Sich über Lust und Unlust an einem Text auszutauschen, ist der direkteste Weg, mitten in den Streit der Interpretationen zu geraten.

IV.

"Nun hatten wir ... manchmal Langeweile, wir wollten lesen, und ehe wir's uns versahen, ward unsere lange Weile noch länger. Endlich hatte Philinen den herrlichen Einfall, die sämtlichen Bücher auf einem großen Tisch aufzuschlagen; wir setzten uns gegen einander und lasen gegen einander, und 
immer nur stellenweise, aus einem Buch wie aus dem andern. Das war nun eine rechte Lust!«.

Johann Wolfgang von Goethe, Wilhelm Meisters Lehrjahre (1794-96)

Die genannten Gesichtpunkte liessen sich verfeinern und vermehren, und mit der Zeit erhielte man eine zunehmend präzise Vorstellung von den Faktoren, die einen bestimmten Fall von Unlust am Text angemessen beschreibbar machen. Im Folgenden soll jedoch eine andere Perspektive erprobt werden. Die Aufmerksamkeit gilt den praktischen Konsequenzen einer wie auch immer verursachten, wie auch immer gearteten Unlusterfahrung: Was tut ein Lesender, wenn Unlust aufkommt? Oder genauer: Was tut er stattdessen, nämlich statt seine unlustvolle Lektüre fortzusetzen? Welche Massnahmen ergreift er, um die Störung durch Unlust zu überwinden, und mit welchem Erfolg?

Und hier zeigt sich, im Gegenzug zur fortschreitenden Differenzierung der Unlust selbst, erstaunlicherweise eine relative Homogenität. Die Unlust am Text - dies die These - ist ein Gleichmacher. Der Fülle der Unlüste steht medial bedingt ein eher begrenzter Katalog von Massnahmen gegenüber, um auf sie zu reagieren. So verschieden sie und ihre Anlässe auch sein mögen, so vergleichsweise einheitlich sind die Weisen, ihnen zu begegnen bzw. zu entgehen. An diesem Punkt stehen sich Musil- und Pilcher-, weibliche und männliche, junge und alte, reiche und arme Lesende plötzlich wieder sehr viel näher, und auch die historischen und gattungsmässigen Spezifika verlieren an Bedeutung. Die möglichen, durch die strukturelle Beschaffenheit des Akts des Lesens regulierten Massnahmen lassen sich versuchsweise unter den Stichworten Anderes lesen, Anders-Lesen, Nicht-Lesen gruppieren.

\section{Anderes lesen}

"Wir suchen Schriftsteller, wir suchen Stile, Schluß mit den bloßen Spielkameraden, wir verlangen Lebensgefährten«.

Daniel Pennac, Wie ein Roman (1992)

Die verbreitetste Weise auf eine unlustvolle Lektüreerfahrung zu reagieren, ist vermutlich die, $\mathrm{zu}$ einem anderen Buch $\mathrm{zu}$ greifen, in der Hoffnung, dass dieses den erwünschten Genuss bereite. Nur: zu welchem? Denn auch die Umsteigestrategie garantiert nicht für Erfolg, da - das Grunddilemma wurde eingangs benannt - die Alternativlektüre unter Umständen ebenso zu verwerfen ist wie die ursprünglich gewählte.

Hier empfiehlt sich der Kanon oder auch die >klassische Literatur als Ausweg, der bewährte Bestand >grosser`, ’bedeutsamer`Werke. 
Ihre übergeschichtliche »Sagkraft« (Gadamer) verspricht, wenn keine garantierte, so wenigstens wahrscheinliche Befriedigung. Doch ist keineswegs nur an die `klassischen` (’humanistischen`, ’bildungsbürgerlichen`) Kanones zu denken, sondern auch an schwächere Kanonformen wie den Borchardtschen $>$ Ewigen Vorrat deutscher Poesier (1926), Reich-Ranickis Karton deutscher Romane und die leiser auftretenden >Leseempfehlungen ' germanistischer Seminare, vor allem aber auch an die jeweiligen Gegenkanones und, schlicht, die durch Verkaufszahlen kanonisierten Titel auf den Bestenlisten. Allerdings kann sich sowohl der Griff zum klassischen oder gegenklassischen Klassiker als auch die Entscheidung für den Bestseller als Scheinlösung erweisen, denn gerade hier sieht sich der Lesende gesteigert unter Druck, eine positive Lektüreerfahrung zu machen. Wenn sich beim Lesen so oder anders approbierter suter Literatur eine Unlust bemerkbar macht, liegt nahe (vgl. Anton Reisers $>$ Messias`-Lektüre), dass nicht das Buch, sondern er selbst, der schlechte Lesende, das Problem ist.

Diese Erfahrung führt vielleicht zur vielleicht aussichtsreicheren Lösung, anderes anders zu lesen, nämlich immer dasselbe: Die vielen Bücher und offiziellen Kanones werden durch die wenigen immer wieder (anders) gelesenen eines subjektiven Kanons abgelöst; die extensive wird zur intensiven Lektüre. Ein Beispiel solch fortschreitender Beschränkung bietet Saul Bellows Mr. Sammler:

"Aber nach vier oder fünf Jahren dieser Diät [Sammler liest Kulturgeschichtliches, moderne Philosophen und den `Doktor Faustus] wünschte er nur noch gewisse religiöse Autoren des dreizehnten Jahrhunderts zu lesen, Suso, Tauler und Meister Eckhart. In seinen siebziger Jahren interessierte er sich für wenig mehr als Meister Eckhart und die Bibel ".

Manche Leser/innen ziehen aus enttäuschenden Erfahrungen mit einem Buch eine andere, in gewissem Sinne gegenläufige Konsequenz. Sie beugen der Unlust vor, indem sie - anders als in spielerischer Weise Philine und Friedrich - von vornherein mehrere Bücher gleichzeitig lesen. Norbert Bolz etwa hat kürzlich in einer Auskunft über seine aktuelle Lektüre bemerkt, dass er seit seiner Studentenzeit "das Lesen als eine Art Multi-Tasking« betreibe: "Mehrere Bücher werden >gleichzeitig` gelesen - und zwar bis zu sieben ... Das ist deshalb sinnvoll, weil auch das beste Buch nach spätestens einer Stunde ermüdet ${ }^{10}$. Ein bestimmtes Buch wird vom Erfolgsdruck entlastet, indem es zu einem unter mehreren wird. Befriedigt es nicht, wird es ohnehin bald durch ein anderes abgelöst. Bolz' Begründung des >Buchzapping oder Simultanlesens ist allerdings radikaler, da sie die

\footnotetext{
9 S. Bellow, Mr. Sammlers Planet. Roman, Deutsch von W. Hasenclever, München 1990, 44.

${ }^{10}$ N. Bolz, "Was liest...", Literaturen 5, 2004, 94.
} 
Unlust weniger auf den Text selbst, als auf die interessemässige Ermüdung des Lesenden zurückführt.

Was aber, wenn nichts Anderes gelesen werden kann, weil gerade dies gelesen werden muss, sei es aus schulischem oder beruflichem Zwang, sei es aus kulturbeflissener Selbstverpflichtung? - Dann muss das Gleiche anders gelesen werden.

\section{Anders-lesen}

"Das Buch war grauenhaft langweilig. Ich hatte zwei Lesezeichen darin: das erste zeigte an, wie weit ich schon war, das zweite zeigte mein selbst gestecktes Tagesziel, bis da und da - bis auf Seite 48 - will ich heute durchhalten. Ich hielt nie durch und erreichte mein Tagesziel nicht».

Peter Bichsel, Der Leser. Das Erzählen (1982)

Die anspruchsvollere Alternative zum unlustbedingten Lesen anderer Texte ist das Anders-Lesen. Ersetzt wird in diesem Fall nicht der Text, sondern die Art und Weise, ihn zu lesen.

Einige dieser Les-Arten nähern das Lesen stark dem Schreiben an. Sie steigern den produktiven Anteil des Rezipienten, indem sie den Text in der einen oder andern Weise lesend umschreiben. Was er von sich aus nicht bieten will, wird ihm durch mehr oder weniger aufdringliche Co-Autorschaft eingegeben. Um mit einem extravaganten Fall zu beginnen: Lichtenbergs Praxis des cross-reading. Zur Aufwertung dröger Lokalnachrichten empfiehlt er eine Lektüre, die durch Missachtung des mehrspaltigen Zeitungssatzes einen neuen, lustvoller zu lesenden Text generiert. Dieser lautet dann etwa "Dem Förster $\mathrm{zu}$ Wiesloch ist gestern ein junges Rind entlaufen - um künftigen Sonntag seine Antrittspredigt zu halten«. Oder: »Es wird eine Köchin gesucht, die mit Backwerk umzugehen weiß - zu zwei Personen eingerichtet, nebst etwas Kellerraum «" Der so lesend hervorgebrachte Text ist an sich flüchtig, er erhält sich allein durch die Beschreibung des Lese-Schreib-Verfahrens selbst. Von Anfang an auf Niederschrift angelegt, ist dagegen die Leseweise mancher Anagrammatiker, die ihrer Permutationsmaschinerie einen ungeliebten Text einspeisen, in kritischer Absicht, um ihn neu und besser zu arrangieren (freilich auch, im Sinne einer Hommage, eine besonders geliebte Zeile). Gerhard Rühms eben erschienene, eher belanglose anagrammatische Relektüre des Dekalogs muss wohl in diesem Sinn verstanden werden ${ }^{12}$; in eine vergleichbare Richtung zielen Christian Uetz' produktive Misreadings, die Worten und Sätzen ihren unbewussten Neben- oder Gegensinn ablauschen.

\footnotetext{
${ }^{11}$ G.Ch. Lichtenberg, Schriften und Briefe, Bd. II, (Sudelbücher II, Materialhefte, Tagebücher), hg. von W. Promies, Darmstadt 1971, 161 (= Heft G 139-144).

${ }_{12}$ Vgl. G. Rühm, Was verschweigt die schwarze Witwe? Schrift-, Sprech- und Bildanagramme, Graz 2004, 49-60.
} 
Solche poietischen Weisen des Anders-Lesens sind vermutlich nicht sehr verbreitet. Eine geläufigere Form, der Unlust durch aktiveres Lesen Rechnung zu tragen, besteht darin, einer gleichfalls im Raum befindlichen Person mitzuteilen - oft gegen ihren Willen -, welch bodenlos schlechten Text man im Begriff ist zu lesen, um dann ein negatives Florilegium zum besten zu geben, das in den Hinweis mündet, wie die betreffenden Stellen reigentlich sollten oder, bescheidener, lauten könnten. Steht für diese Form der Unlustverarbeitung kein Hörer zur Verfügung, wird das Lesen möglicherweise schreibend im ganz konkreten Sinn: Durch Marginalien

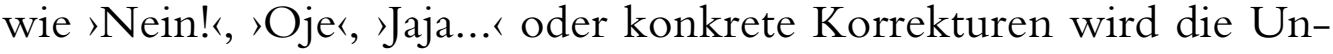
lust am Text dem Text selbst eingeschrieben. Die öffentlich wahrnehmbare Gestalt dieser Formen, anders zu lesen, ist die Literaturkritik. Der Kritiker schreibt im Verlauf der unlustvollen Lektüre bereits lustvoll an seinem Verriss, er nimmt sich, mit Benjamin gesprochen, das Buch "so liebevoll vor, wie eine Kannibale sich einen Säugling zurüstet $\aleph^{13}$.

$\mathrm{Zu}$ nennen bleiben die populärsten Formen des Anders-Lesens, zunächst das selektive und das >diagonale Lesen. Sie zielen weder auf lesende Umschreibung noch auf die Hervorbringung kritischer Metatexte, sondern auf eine Rationalisierung der Lektüre: Wenn der Text sich schon nicht gut liest, will man ihn schnell hinter sich bringen bzw. schnell die Stellen finden, die eine eingehende Lektüre lohnen. Auch diese Strategien haben allerdings ihre Tücken, auch sie eignen sich nur in bestimmten Fällen zur Wiederherstellung des lustvollen Lesens. Das selektive Lesen, das Blättern und Überspringen einzelner Passagen, riskiert, Entscheidendes - auch Lustvolles $\mathrm{zu}$ verpassen und wegen fehlender Informationen (über Entwicklungen im Handlungsverlauf etwa) bestimmte Pointen zu überlesen. Das Gleich gilt grano cum salis auch für das diagonale Lesen. Auch der eiligen oberflächlichen Lektüre entgehen eher die Finessen, auf die es vielleicht ankäme. (Beide, die diagonale und die selektive Lektüre, kommen aus Zeitmangel oder anderen Gründen freilich nicht erst als Kompensationsmodus zum Zuge, sondern bereits bei der sneutralen Erstbegegnung. Im Falle von wissenschaftlichen oder Sachpublikationen wird zudem oft von vornherein nicht das Ideal der integralen Lektüre angestrebt. Die entsprechenden Texte sind auf partielle Rezeption angelegt, indem sie durch gliedernde Massnahmen - Kapitelüberschriften, Inhaltverzeichnis, Register verschiedenster Art - die Auffindung bestimmer Passagen erleichtern. Auch als Kompensationsmassnahme ist die selektive Lektüre daher bei

\footnotetext{
${ }^{13}$ W. Benjamin, Einbahnstraße (Die Technik des Kritikers in dreizehn Thesen, These X), in: ders., Gesammelte Schriften (= GS), unter Mitwirkung von Th.W. Adorno und G. Scholem hg. von R. Tiedemann und H. Schweppenhäuser, Bd. IV/1 (Kleine Prosa, BaudelaireÜbertragungen), Frankfurt a.M. 1972, 108.
} 
wissenschaftlichen Publikationen eher erfolgreich als im Fall meist weniger bzw. weniger vordergründig strukturierter literarischer Texte).

Das diagonale und das selektive Lesen verweisen darauf, dass eine verbreitete Ursache der Unlust am Text bereits in der Überforderung durch dessen blossen Umfang liegt. Daher wird man - divide et impera - das zu Lesende überschaubar halten, ganz im Sinne der binsenwahren Lebensweisheit, nicht das Ganze, sondern nur den unmittelbar nächsten Schritt ins Auge zu fassen. Die zitierte Lesezeichen-Technik Peter Bichsels mag als Beipiel für eine derartige Portionierung oder Parzellierung der Lektüre dienen, als negatives, da Elisabeth Müllers >Christeli<, von dem an besagter Stelle die Rede ist, dem jungen Peter, dessen Orthographie sich durch regelmässiges Lesen verbessern soll, selbst in homöopathischen Dosen unerträglich bleibt. ${ }^{14}$

Ferner das Rückwärts-Lesen, d. h. das erneute Lesen eines eben mit negativem Ergebnis gelesenen Satzes oder Textes. Wer so liest, realisiert das Principle of Charity; er geht davon aus, dass die erfahrene Unlust sich möglicherweise weniger dem Text als vielmehr der eigenen unzulänglichen Rezeption verdankt. Durch erneutes Lesen, sei es punktuell oder integral, wird dem Text die Chance eingeräumt, die erste Lektüreerfahrung zu widerlegen. Anders als bei der diagonalen und selektiven Lektüre soll nicht eine Beschleunigung, sondern eine Verlangsamung des Lesens zur Befriedigung verhelfen, im Extremfall das mehrfache, immer wieder neu ansetzende SichVorarbeiten von Satz zu Satz. Nicht in jedem Fall wird der Lesende dazu bereit sein, am ehesten wohl dann, wenn sich die Lektüre des betreffenden Textes aus bestimmten Gründen (wie Kanonzugehörigkeit oder warme Empfehlung durch verlässliche Bekannte) mit positiven Erwartungen verbindet.

Eine Variante des Rückwärts-Lesens ist das trotzige DennochWeiterlesen. In diesem Fall investiert der Lesende seine charity nicht in eine erneute Lektüre, die die erhoffte Wende herbeiführen könnte, sondern in ein Durchhalten, wenn gefühlsmässig an sich deren Abbruch angezeigt wäre. Er relativiert die Unlust durch die Vorstellung, dass man sich in bestimmte Bücher eben erst mühsam >hineinlesen müsse, bis an den Punkt, da man deren Ton oder Sinn erfasse bzw. diese einen, und die Mühsal in Labsal umschlage, vergleichbar den ersehnten Endorphinausschüttungen des Langstreckenläufers. Um nicht doppelt betrogen zu sein, falls dieser Umschlag ausbleibt, wird das Dennoch-Weiterlesen möglicherweise limitiert bis Seite X - und damit riskiert, dass er auf Seite $\mathrm{X}+1$ vielleicht erfolgt wäre.

${ }^{14}$ Vgl. P. Bichsel, Der Leser. Das Erzählen, Frankfurter Poetik-Vorlesungen, Darmstadt 1982, 35 . 
$\mathrm{Zu}$ diesen Massnahmen, die den Vollzug des Lesens im engeren Sinn betreffen, treten schliesslich eine Reihe summarisch zu nennender lustfördender Manipulationen an der Leseszene in einem äusserlichen Sinn: die Optimierung des Leseambientes durch die Einnahme angenehmer Haltungen oder Getränke, die Stabilisierung der angeschlagenen Motivation durch eine in Aussicht gestellte Belohnung (einen Krimi etwa), usw.

\section{Nicht-lesen}

"Daher ist ... die Kunst nicht zu lesen, höchst wichtig».

Arthur Schopenhauer, Über Lesen und Bücher (1851)

Das Stichwort des Nicht-Lesens bedarf der Ergänzung, denn es benennt lediglich, dass die Lektüre abgebrochen wird, lässt aber offen, was an ihre Stelle tritt. Worum es geht, sind Fälle von Nicht-Lesen und ..., nämlich Nicht-Lesen und ... einer beliebigen anderen Tätigkeit nachgehen. - Der Regelfall des Nicht-Lesens ist vermutlich die Ersetzung des Lesens durch eine beliebige andere Tätigkeit wie radfahren, rasenmähen und dergleichen. $\mathrm{Ob}$ diese Formen des Stattdessen befriedigender sind als die unbefriedigende Lektüre ist allerdings ungewiss, denn eigentlich wollte man ja lesen und nicht rasenmähen.

... selbst schreiben. - Bücher entstehen aus der Unlust, aus dem Ungenügen an bestehenden Büchern: „Schriftsteller», so Benjamin, "sind eigentlich Leute, die Bücher nicht aus Armut sondern aus Unzufriedenheit mit den Büchern schreiben, welche sie kaufen könnten, und die ihnen nicht gefallen ${ }^{15}$. Diese Unzufriedenheit rührt nun aber, so lässt sich mit Harold Bloom differenzieren, nicht nur daher, dass das betreffende Buch zu schlecht wäre, sondern, im Gegenteil, zu gut. Um selbst zu Ruhm zu gelangen, muss der Autor seine Vorgänger (Väter), denen er in ödipaler Hassliebe verpflichtet ist, überwinden (ermorden). Seine "Anxiety of Influence führt ihn zu willkürlich-destruktiven "Misreadings", die als Rückverweise in seinen eigenen Text eingehen. Die Unlust, die aus Bewunderung und einem folgedessen gesteigerten Willen zur Selbstbehauptung resultiert, schreibt mit, wenn das Nicht-Lesen ins Schreiben mündet.

... das angelesene Buch schänden. - Die drastischste Variante des Nicht-Lesens ist die Bücherschändung. Der Bücherschänder lässt das Lesen, nicht aber das Buch, das er "zerstört, indem er [ihm] Seiten, Bilder, Tafeln o.ä. entnimmt ${ }^{16}$. Um Rache zu nehmen für den un-

\footnotetext{
${ }^{15}$ W. Benjamin, Denkbilder (Ich packe meine Bibliothek aus: Eine Rede über das Sammeln), in: GS IV/1, 390.

${ }^{16}$ M. Rehm, Lexikon Buch, Bibliothek, Neue Medien, München 1991, 59. Rehm bemüht sich sorgsam um eine definitorische Scheidung des Bücherschänders vom Biblioklasten (vgl. ebd., 29), der ebendies als Sammelleidenschaft tut, und insofern die kriminelle Variante des
} 
lustvollen Gehalt wird die Körperlichkeit des Buches - vielleicht lustvoll - malträtiert. Die Bücherschändung stellt die unkultivierte oder aber radikalisierte Variante des hämischen Annotierens dar.

... behaupten, gelesen zu haben. - In Zusammenhängen unfreiwilligen und deshalb unlustanfälligeren Lesens kann die Unlust am Text so massiv sein, dass die Lektüre unterlassen und stattdessen einfach behauptet wird. Zur Vermeidung der Unlust wird zur Lüge Zuflucht genommen bzw. in Kauf, dass diese zu gleichfalls unlustvollen Sanktionen führen könnte.

... schlafen. - Die Ersetzung des Lesens durch das Schlafen kann nur unter Vorbehalten als Variante gelten, da das Einnicken über einem Buch nicht unbedingt Folge einer Unlustregung sein muss, sondern schlicht auch Ausdruck allgemeiner körperlicher Erschöpfung. Anzunehmen ist wenigstens, dass die betreffende Lektüre wenn nicht wirklich unlustvoll so auch nicht wirklich lustvoll war. Denn der begeisterte Lesende kann, wie das Beispiel von Vater und Sohn Rousseau zeigt, »ohne Unterbrechung ... die Nächte damit ver[bringen] «: »Wir konnten niemals aufhören, ehe das Buch nicht zu Ende war. Manchmal, wenn wir am Morgen die Schwalben hörten, sagte mein Vater ganz beschämt: >Wir wollen schlafen gehen! Ich bin ein viel größeres Kind als du « ${ }^{17}$.

Wenn Schopenhauer auf die höchstwichtige »Kunst ... nicht zu lesen« verweist, hat er freilich etwas anderes im Sinn als die genannten Kompensationshandlungen. Seine Mahnung gilt dem rechten Unterscheiden, der Vermeidung des Nicht-Lesenswerten: »Vom Schlechten kann man nie zu wenig und das Gute nie zu oft lesen.... Um das Gute zu lesen, ist eine Bedingung, dass man das Schlechte nicht lese: denn das Leben ist kurz, Zeit und Kräfte beschränkt « ${ }^{18}$. Seine Auskünfte darüber, wie denn das eine vom anderen zu unterscheiden sei, sind grossartig gerade in ihrer kompromisslosen und polemischen Einseitigkeit. Es gilt die simple Regel: alt = gut, neu = schlecht; zu lesen, ausschliesslich, sind »die Gedanken de[r] großen Geist[er] « der »Vorzeit", auf keinen Fall jedoch »das seichte, fade Geträtsche eines heutigen Flachkopfs «" ${ }^{19}$, das wie "wuchernde[s] Unkraut ..." die "guten Bücher ... und ihre ... edelen Zwecke ${ }^{20}$ erstikke.

Bibliomanen (vgl. ebd.) darstellt, der, pflegt er seine Leidenschaft im Geheimen, als Bibliotaph (vgl. ebd., 30) zu bezeichnen ist.

17 J.-J. Rousseau, Bekenntnisse (1781), aus dem Französischen von E. Hardt, mit einer Einführung von W. Krauss, Frankfurt a.M. 1985, 41.

18 A. Schopenhauer, Über Lesen und Bücher, in: ders., Parerga und Paralipomena: kleine philosophische Schriften, Bd. 2 (= Sämtliche Werke, Bd. 6), Mannheim 1988, 592.

19 Ebd.

20 Ebd., 591. 
Peter Bichsels >Lob des Nichtlesers` (1993) macht dagegen auf eine letzte bedenkenswerte Spielart des Nicht-Lesens aufmerksam: »Der Briefwechsel zwischen Robert und Clara Schumann. Ein wunderbares Buch - nur habe ich es noch nicht gelesen ${ }^{21}$. NichtLesen in diesem Sinn ist präventives Nicht-Lesen. Es folgt nicht der Unlusterfahrung, es geht ihr, zu ihrer Vermeidung, voran. Die "potentielle Lektüre" (Schlaffer) beugt möglicher Unlust vor, indem sie es beim Besitz eines - vermutlich grossartigen - Werks bewenden lässt; der Verzicht auf die Lektüre ist der Preis für eine intakte und daher lustvolle Erwartungshaltung. Philipp Swallow, Professor für englische Literatur und Protagonist des Campusromans >Changing Places (1975) des Professors für englische Literatur David Lodge, pflegt auf jede seiner Reisen die >Divina Comedia mitzunehmen, um sie jedesmal zwar ungelesen, doch auch unvermindert vielversprechend wieder nach Hause zu tragen.

V.

Die Hinweise auf eher exzentrische Ausprägungen bestimmter Kompensationsmodi machen deutlich, wie eingeschränkt und normiert deren Repertoire ist, eingeschränkt gemessen an der Spezifität und Individualität einer bestimmten Unlust-Szene. Die Unlust am Text ist ein Gleichmacher. Das gilt auch dann, wenn die abstrahiert beschriebenen Strategien im faktischen Vollzug des Lesens in Kombinationen, Mischformen und individuellen Ausprägungen auftreten.

Der gewählte Zugriff, das ist vielleicht seine Schwäche, bestätigt zwar nicht den common sense, dass die Lust die Regel ist, folgt aber, indem er sich an den Kompensations- oder Reparaturstrategien orientiert, der These, dass sie es sein soll. Daraus ergibt sich ein wenigstens zweifacher Bedarf nach Vertiefung. Einerseits wären die Phänomene und Begriffe von Lust und Unlust präziser zu fassen, die Übergänge zwischen beiden und deren Ineinander; andererseits wäre im Sinne einer Gegenprobe auf die Gegenprobe zu fragen, wie sich die erhobenen Massnahmen zur Lust am Text verhalten. Denn wenigstens manche von ihnen kommen, wie bemerkt, nicht nur als Kompensations-, sondern auch als Normalmodus zur Anwendung, vor allem aber dienen einige ebenso der Eliminierung der Unlust wie der Intensivierung oder Wiederholung der Lust: das retardierende Rückwärts-Lesen, die Formen des schreibenden Lesens und auch das Nicht-Lesen, insofern es sich ein Stück Lusttext für später aufhebt.

\footnotetext{
${ }^{21}$ P. Bichsel, Lob des Nichtlesers. Rede zum zehnten Jubiläum der Huss'schen Universitätsbuchhandlung in Frankfurt am Main (1993), in: ders., Das süsse Gift der Buchstaben. Reden zur Literatur, Frankfurt a.M. 2004, 77.
} 
Nachzudenken über die Unlust am Text kann nicht zum Ziel haben, was weder wünschenswert noch möglich ist: diese zu eliminieren. Die genannten Strategien sind allesamt unzuverlässig; Lesen bleibt ein Vollzug mit offenem Ausgang, allem voran aus dem eingangs genannten strukturellen Grund. Eine gewisse Aufmerksamkeit für die Störungen im Lesefluss und deren Konsequenzen macht aber sensibel für das, was ihn im - supponierten - Regelfall in Gang hält. Insofern die Unlust am Text dazu nötigt, Anderes, anders oder nicht zu lesen, zeigt sich an ihr, wie gelesen wird, welches Lesen enttäuscht wurde. Und das kann, bestenfalls, dazu verhelfen, besser zu lesen, was auch heisst: besseres Anderes, besser anders und besser nicht.

Doch muss die Unlust am Text bereits in viel schlichterer Weise geehrt werden, denn immer wieder schlägt die Unlust des einen wunderbar um in Lust für den anderen. Die Geschichte der Zensur genannten staatlichen oder römisch-katholischen Unlust an Texten ist keineswegs nur tragisch, sondern, wie etwa Peter Godmans Arbeiten über den Index Librorum Prohibitorum zeigen ${ }^{22}$, auch durchaus amüsant, und glänzende Verrisse sind ebenso eine Labsal für den Lesenden wie Kollegenschelten. ${ }^{23}$ Die Folgende, sie stammt von Franz Kafka, mag als Beispiel dienen, als Beispiel, das zudem anregen mag, einen bislang unerwähnten Schauplatz verbreiteter Unlust am Text zu erkunden, die literarische Lesung:

"27. November [1910]. Bernhard Kellermann hat vorgelesen. `Einiges Ungedrucktes aus meiner Feder, so fing er an. Scheinbar ein lieber Mensch, fast graues, stehendes Haar, mit Mühe glatt rasiert, spitze Nase, über die Backenknochen geht das Wangenfleisch oft wie eine Welle auf und ab. Er ist ein mittelmäßiger Schriftsteller mit guten Stellen (ein Mann geht auf den Korridor hinaus, hustet und sieht umher, ob niemand da ist), auch ein ehrlicher Mensch, der lesen will, was er versprochen hat, aber das Publikum ließ ihn nicht, aus Schrecken über die erste Nervenheilanstaltsgeschichte, aus Langeweile über die Art des Vorlesens gingen die Leute trotz schlechter Spannungen der Geschichte immerfort einzeln weg mit einem Eifer, als ob nebenan gelesen werde. Als er nach dem ersten Drittel der Geschichte ein wenig Mineralwasser trank, ging eine ganze Menge Leute weg. Er erschrak. 'Es ist gleich fertig, log er einfach. Als er fertig wurde, stand alles auf, es gab etwas Beifall, der so klang, als wäre mitten unter allen den stehenden Menschen einer sitzen geblieben und klatschte für sich. Nun wollte aber Kellermann noch weiterlesen, eine andere Geschichte, vielleicht noch mehrere. Gegen den Aufbruch öffnete er nur den Mund. Endlich, nachdem er beraten worden war, sagte er: \Ich möchte noch gerne ein kleines Märchen vorlesen, das nur fünfzehn Minuten dauert. Ich mache fünf Minuten Pause . Einige

\footnotetext{
${ }^{22}$ Vgl. P. Godman, Die geheime Inquisition. Aus den verbotenen Archiven des Vatikans, München 2001; P. Godman/J. Brandt, Weltliteratur auf dem Index. Die geheimen Gutachten des Vatikans, München 2001.

${ }^{23}$ J. Drews (Hg.), Dichter beschimpfen Dichter. Die ultimative Sammlung aller Kollegenschelten, Leipzig 1994.
} 
blieben noch, worauf er ein Märchen vorlas, das Stellen hatte, die jeden berechtigt hätten, von der äußersten Stelle des Saales mitten durch und über alle Zuhörer hinauszurennen « ${ }^{24}$.

- Andreas Mauz ist Stipendiat am Institut für Hermeneutik und Religionsphilosophie.

${ }^{24}$ F. Kafka, Tagebücher 1910-1923, Frankfurt a.M. 1983, 21 . 